\title{
On the Ergodic Properties of Nowhere Dispersing Billiards
}

\author{
L. A. Bunimovich \\ P. P. Shirshov Institute of Oceanology, Academy of Sciences USSR, Moscow 117218, USSR
}

\begin{abstract}
For billiards in two dimensional domains with boundaries containing only focusing and neutral regular components and satisfacting some geometrical conditions B-property is proved. Some examples of three and more dimensional domains with billiards obeying this property are also considered.
\end{abstract}

\section{Introduction}

In the present paper dynamical systems with elastic reflections, usually called billiards, are studied. From the point of the general theory of dynamical systems a billiard can be considered as a geodesic flow on a manifold with a boundary. Billiards give models for many problems of classical mechanics, statistical physics, optics, and acoustics.

The statistical properties of one particular class of billiards were studied by Sinai [1] who called them dispersing. The simplest example of such billiards is the motion of a material point on a torus with elastic reflections from fixed spherical particles. It was shown in [1] that the corresponding dynamical system is $K$ system. In [2] this result was generalized to a wider class of dispersing billiards.

An intuitive explanation of the analogy between dispersing billiards and goedesic flows in spaces of negative curvature is due to Arnold [3]. The exponential expansion of trajectories, which is due in geodesic flows to negative curvature, follows from collisions with the convex (from inside) components of the boundary of the domain.

Hopf [4] established that a geodesic flow on a surface with the gaussian curvature taking the values of different signs is ergodic if every trajectory spends most of the time in the regions with negative curvature. The analogous result has been proved [5] for domains with boundaries containing both dispersing and focusing components.

In accordance with the traditional ideas stochastic properties of the billiard follow from the scattering of trajectories which results from collisions with the boundary. If small focusing parts of the boundary are also present and the scattering is sufficiently large then the stochasticity is conserved. However, it is 
known that the billiard system inside the domain bounded by a smooth plane convex curve has a set of positive measure consisting of the tori $[6,7]$. Thus the dynamical system generated by this billiard is obviously nonergodic.

In [8] it was proved that there exist convex domains on the plane whose boundaries contain no focusing components at all, and nevertheless they generate billiards which are ergodic and have the $K$-property. A necessary condition for ergodicity in [8] was the presence of straight segments in the boundary. The stochastic properties of such billiards arise of a consequence of successive noncoordinate focusing of local small bundles of trajectories when they reflect of the boundary. Such bundles contract after reflection from the focusing component of the boundary, then it goes through conjugate point, after which it expands and has time enough to expand sufficiently before the following reflection of the boundary occurs.

In the given paper a general theorem is proved, which states sufficient conditions for $K$-property (and $B$-property) for regions whose boundary has no dispersing components. Some examples of application of this theorem to concrete classes of domains are given (in particular for domains with boundaries possessing only focusing components). Possible generalizations of this theorem for billiards in multidimensional (nonplane) domains are considered.

\section{Description of the Class of Domains Under Consideration and Main Properties of Billiards}

Let $Q$ be a closed connected domain on the Euclidean plane or on a two dimensional torus with Euclidean metric. We assume that the boundary $\partial Q$ consists of a finite number of smooth nonselfintersected curves (of class $C^{3}$ ) intersecting only at their ends. A single curve of this collection will be called a regular component of the boundary and will be denoted by $\partial Q_{i}(i=1, \ldots, k)$. The points of intersection of regular components will be called singular boundary points. The boundary $\partial Q$ equipped with a field of inward (with respect to $Q$ ) normals $n(q)$, where $q \in Q$. The angle $\varphi$ is measured with respect to $n(q)$. Let us denote by $\partial Q^{+}$the collection of all regular components of the boundary with positive curvature (w.r. to the chosen direction of the normal) in every point. Analogously $\partial Q^{-}$is the collection of all regular components with negative curvature and $\partial Q^{0}$ is the collection of all regular components with curvature zero. It is assumed that the curvature of every regular component has a constant sign and is either different from zero or equal to zero in all its points. We shall call $\partial Q^{+}, \partial Q^{-}$, and $\partial Q^{0}$ dispersing, focusing and neutral part of the boundary respectively.

Let $M$ be the restriction of the unit tangent bundle of the plane (or torus) to $Q$. Points $x=(q, \mathbf{v})$ of the space $M$ will be called line elements and $q \in Q$ - the support of line element $x=(q, \mathbf{v})$. We denote the natural projection of $M$ onto $Q$ by $\pi$. It is clear that $M$ is also a manifold with boundary. The boundary $\partial M$ is formed of those line elements such that $\pi(x) \in \partial Q$. We define the regular components of the boundary $\partial M$ as $\partial M_{i}=\pi^{-1}\left(\partial Q_{i}\right)$. On $\partial M_{i}$ we introduce a natural coordinate system $(r, \varphi)$ where $r$ is the parameter of arc length along $\partial Q_{i}$ and $\varphi$ an angular parameter. 
By billiard in $Q$ we mean a dynamical system in $M$, generated by the motion of a tangent vector $x$ in $M$ along a geodesic line with unit speed. When a line element $x$ reaches the boundary of the domain $Q$ an instanteneous reflection from $\partial Q$ takes place according to the rule "the angle of incidence is equal to the angle of reflection", i.e. the component of the vector tangential to $\partial Q$ is preserved and the normal component changes sign, after that the motion inside $Q$ is continue along a geodesic line. If a trajectory hits a singular point of the boundary we shall consider that the following motion is not defined.

Let $S_{-}=\{x \in \partial M:(x, n(q)) \leqq 0, q=\pi(x)\}$, where $(x, y)$-scalar product of line elements $x$ and $y$. The set

$$
S=S_{1} \cup S_{2}=\{x \in \partial M:(x, n(q))=0, q=\pi(x)\} \cup\left\{x \in \partial M: \pi(x) \in \partial Q_{i} \cap \partial Q_{j}\right\},
$$

which consists of line elements tangent to the boundary or have support in singular points of the boundary. $S$ will be called the set of singular points of the boundary.

In the space $M$ we introduce a measure $\mu$ by letting $d \mu=d q d \omega$, where $d q$ is the measure induced on $Q$ by the Euclidian metric and $d \omega$ is the natural measure on the unit circle $S^{1}(q)$. The one-parameter group of shifts along the orbit of the billiard will be denoted by $\left\{S_{t}\right\},-\infty<t<+\infty$. It is obvious that it preserves the measure $\mu$. Therefore $\left\{S_{t}\right\}$ is a flow in the sense of ergodic theory.

Let $\mathscr{U}_{1}=S_{-} \backslash S$ and let $\tau(x)$ be the nearest negative moment of reflection of the orbit of point $x$ from the boundary. It is easy to see that $\tau(x)>-\infty$ and that for any $x \in \mathscr{U}_{1}$ there is a well defined mapping $T x=S_{\tau(x)-0} x \in \mathscr{U}_{1}$. The mapping $T$ is discontinuous. It has singularities on the set $\mathscr{U}_{2}=T^{-1} \mathrm{~S} \cup \mathrm{S}$.

In $\mathscr{U}_{1}$ we introduce a measure $v$ by letting for any $\mathscr{A} \subset \mathscr{U}_{1}$

$$
v(\mathscr{A})=\int_{\tilde{\mathscr{A}}} \frac{d \mu(y)}{F(y)},
$$

where $\tilde{\mathscr{A}}=\left\{y \in M: y=S_{t} x\right.$ for $\left.0 \geqq t>\tau(x), x \in \mathscr{A}\right\}, F(y)=\tau(x)$. It is not hard to see that $d v(r, \varphi)=-$ const $\cos \varphi d r d \varphi$, where by const we denote the normalization constant. The transformation Tpreserves the measure $v$ and is the derived automorphism of the flow $\left\{S_{t}\right\}$ (see [9]). It is easy to see that (both for $\mu$ and for $v$ ) the measure of trajectories containing singular points of the boundary of the domain $Q$ equals zero. Consequently from now on we shall consider trajectories which contain only regular points of the boundary, since in ergodic theory one can neglect a set of measure zero. It is not difficult to see that ergodicity of the flow $\left\{S_{t}\right\}$ follows from ergodicity of $T$.

In the present paper we shall suppose that $\partial Q^{+}=\emptyset$, i.e. the boundary $\partial Q$ does not contain dispersing components. It is assumed also that $\partial Q$ contains at least one focusing component, i.e. the domain $Q$ is not a polygon. (It is well known $[10,11]$ that the entropy of billiards in polygons equals zero.)

For domains studied by us the following two conditions are fulfilled:

1. The curvature of each focusing component $\Gamma_{i}^{F}\left(i=1,2, \ldots, n_{F}\right)$ of the boundary $\partial Q$ is constant, i.e. $\Gamma_{i}^{F}$ is an arc of some circle $O_{\Gamma_{i}^{F}}$.

2. Let $\mathscr{U}_{1}^{-}=\left\{x: \pi(x) \in \partial Q^{-}\right\}$and $\bar{\tau}(x)$ be the nearest negative moment of reflection from the focusing part of the boundary of the domain $Q$ of the trajectory of the point $x$. Then the length of the chord of the circle $O_{I_{F} F}$ defined by the line 

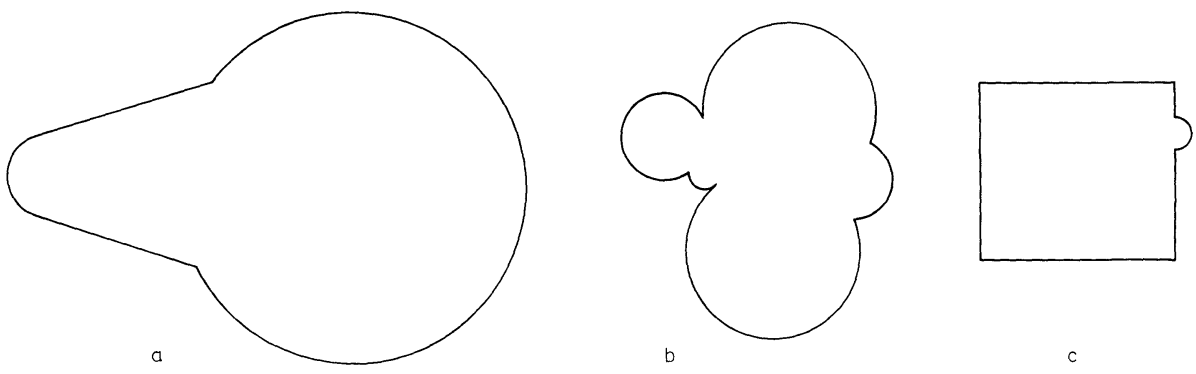

Fig. 1

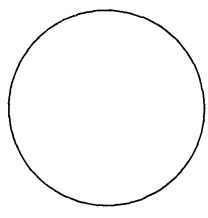

a

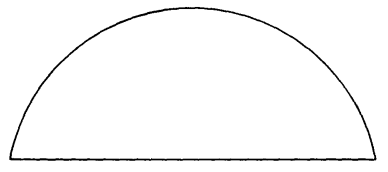

b

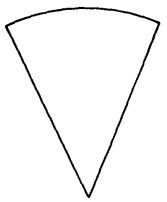

c

Fig. 2

element $x$ is less or equal to $|\bar{\tau}(x)|$. The rigorous inequality is tulfilled on the set $\tilde{U}_{1}^{-}$ of positive measure (with respect to measure $v$ ).

One must mention that the conditions we introduced include the case, when the circles $O_{\Gamma_{i}^{F}}$ and $O_{I_{j}^{F}}$ coincide for $i \neq j$. It is obvious that the class of domains satisfying our conditions is sufficiently larger than that considered in [8].

Examples of domains for which our conditions are fulfilled are shown in Fig. 1; in Fig. 2 we show domains for which they are not fulfilled.

We remind that a dynamical system with discrete time, generated by a transformation $T$ with an invariant measure is called a $B$-system if $T$ is metrically isomorphic to a Bernoully shift. It is obvious that a $B$-system is also a $K$-system. In a recent paper [12] Ornstein has shown that inverse statement is false.

Consider for every natural number $n$ the set $A_{n}^{\alpha}=\left\{x: \pi(x) \in \partial Q, \pi\left(T^{i} x\right) \notin \tilde{\mathscr{U}}_{1}^{-}\right.$for $\left.0<i<n^{\alpha}\right\}$, where $\alpha>0$.

The following main result is true.

Theorem. If a domain $Q$ satisfies conditions 1,2 and there exists a positive number $\alpha<1$ such that the series $\sum_{n=1}^{\infty} v\left(A_{n}^{\alpha}\right)$ is convergent, the billiard inside $Q$ is a B-system.

Let $d$ be the distance on $\mathscr{U}_{1}$ generated by the metric $d s^{2}=d r^{2}+d \varphi^{2}$. We set $d(x)$ $=d\left(x, S_{1}\right)$, and by $\varphi(x)$ we denote an angle between $x$ and $n(q)$, where $q=\pi(x)$. In complete analogy with the case of dispersing billiards (see [1], pp. 147-148) one can prove the following inegualities:

$$
|\cos \varphi(x)| \geqq C_{1} d(x), C_{2}^{\prime \prime} d(x) \leqq|\tau(x)| \leqq \frac{C_{2}^{\prime}}{d(x)},|\cos \varphi(T x)| \geqq C_{3} d(x) .
$$


Consider a curve $\tilde{\gamma} \subset Q$ of class $C^{2}$. By an equipment $\gamma$ of the curve $\tilde{\gamma}$ is meant a continuous section of the tangent bundle over $\tilde{\gamma}$ such that at each point $q \in \tilde{\gamma}$ the line element is orthogonal to the line element tangent to $\tilde{\gamma}$ at the point $q$. Each curve $\tilde{\gamma}$ has two equipments. After an equipment is chosen, one can speak of the curvature of the curve $\tilde{\gamma}$ with sign. If the curvature does not vanish at any point of the curve, then the indication of its value at a single point uniquely determines the equipment.

Let $\tilde{\gamma} \subset Q$ be any smooth curve without selfintersections and let $\gamma$ be its equipment. We assume that $\tilde{\gamma}$ with the equipment $\gamma$ has strictly negative curvature. We assume, further, that on $\tilde{\gamma}$ the parameter of arc length $s$ has been chosen, measured from some interior point. We can also consider $s$ as the parameter describing $\gamma$. We denote by $k(s)$ the curvature of the curve $\tilde{\gamma}$ at the point with coordinate $s$. The equation $k=k(s)$ is the natural equation of the curve $\tilde{\gamma}$.

The following three lemmas can be easily proved with the help of elementary geometrical considerations, the corresponding proofs can be found for instance in [1] (see also [2], where some corrections and specifications are given).

We choose a $t<0$ such that during the time from $t$ to 0 no point $x \in \gamma$ reaches the boundary.

Lemma 1. The natural equation of the curve $\tilde{\gamma}_{1}=\pi\left(\gamma_{1}\right), \gamma_{1}=S_{t} \gamma$ has the form $k_{1}\left(s_{1}\right)$ $=k(s) /(1+t k(s))$, where $s_{1}$ is the parameter of arc length $\tilde{\gamma}_{1}$, which is naturally related to the parameter s on $\tilde{\gamma}$, and $d s_{1} / d s=1+t k(s)$.

We assume now that from time $t$ to 0 each point of the curve $\gamma$ has exactly one reflection from the boundary and that this reflection arises from one and the same regular component of the boundary for all $x \in \gamma$. It follows that $\gamma_{1}=S_{t} \gamma$ is a curve lying inside $M$. For the point with coordinate $s$ we denote by $t(s), \varphi(s)$ and $k^{(0)}(s)$ the moment of reflection from the boundary, the angle of reflection and the curvature of the boundary at the point of reflection, respectively.

Lemma 2. The natural equation of the curve $\tilde{\gamma}_{1}=\pi\left(S_{t} \gamma\right)$ has the form

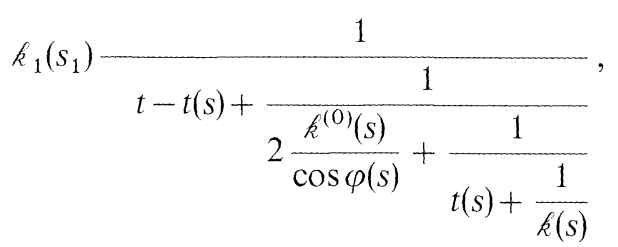

where $\frac{d s_{1}}{d s}=1+t k(s)+2 \frac{k^{(0)}(s)}{\cos \varphi(s)}(1+t(s) k(s))(t-t(s))$.

Having the curve $\gamma$, which appears in Lemma 2, we consider for each point $x(s) \in \gamma$ the least $t(s)>0$ such that $S_{t(s)+0} x(s) \in \mathscr{U}_{1}$, and we assume that $\gamma_{1}=\left\{S_{t(s)+0} x(s)\right\}$ is a curve on the boundary $\mathscr{U}_{1}$ of $M$ belonging to a single regular component. Suppose, further, that in the system of coordinates $(r, \varphi)$ the curve $\gamma_{1}$ is described by the function $\varphi=\varphi(r)$. From Lemma 1 it follows that $\frac{d \varphi}{d r}=\frac{k(s) \cos \varphi(s)}{1+t(s) k(s)}$, where $s$ and $r$ are connected by the relation $\pi\left(S_{t(s)} x(s)\right)=r$.

The following lemma is easily obtained from Lemmas 1 and 2. 
Lemma 3. Let the curve $\gamma_{1} \subset \mathscr{U}_{1}$ be given by the equation $\varphi=\varphi(r)$ and $T \mid \gamma_{1}$ be a smooth transformation. Then $\gamma_{1}^{\prime}=T \gamma_{1}$ is a curve on $\mathscr{U}_{1}$. If the curve $\gamma_{1}^{\prime}$ is given by the function $\varphi^{\prime}=\varphi^{\prime}\left(r^{\prime}\right)$, where the parameters $r$ and $r^{\prime}$ are naturally related, then

$$
\begin{aligned}
& \frac{d \varphi^{\prime}}{d r^{\prime}}=-k^{(0)}\left(r^{\prime}\right)-\frac{\cos \varphi^{\prime}}{\tau(r)-\frac{\cos \varphi}{\frac{d \varphi}{d r}-k^{(0)}(r)}}, \\
& \frac{d r^{\prime}}{d r}=-\frac{\cos \varphi(r)}{\cos \varphi^{\prime}\left(r^{\prime}\right)}\left[1-\frac{\tau(r)}{\cos \varphi(r)}\left(\frac{d \varphi}{d r}-k^{(0)}(r)\right)\right] .
\end{aligned}
$$

From the preceding Lemmas it follows, in particular, that a bundle of parallel trajectories after reflection from the boundary remains parallel, if the reflection took place on a neutral component and is dispersed or focused if the reflection takes place from a dispersing or focusing component respectively.

\section{Some Properties of Series of Consecutive Reflections from the Boundary}

Let the moments of consecutive reflections of the trajectory of the point $x$ from the boundary be $0 \leqq t_{1}<t_{2}<\ldots$. The interval between two successive reflections is $\tau_{i}=t_{i}-t_{i-1}\left(i=1,2, \ldots, t_{0}=0\right)$. We denote by $k_{i}^{(0)}(x)$ the curvature of the boundary at the point of the $i$-th reflection, and by $\varphi_{i}(x)$ the angle of incidence of the $i$-th reflection, $\pi / 2 \leqq \varphi_{i}(x) \leqq 3 \pi / 2$.

Consider for each point $x \in M$ the infinite continued fraction

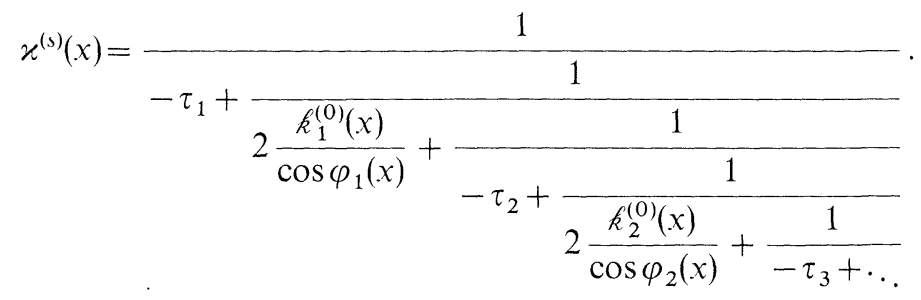

With the help of function $x^{(s)}(x)$ one can construct almost everywhere in the case of dispersing billiards local orthogonal manifolds to the bundles of asymptotic trajectories. These manifolds play a central role in exploring the ergodic properties of billiards. In the following we shall show that analogous manifolds exist also for billiards of the class studied in the present paper.

Somewhat later we shall study the question of convergence for this continued fraction. Until then we shall consider (1) as a formal expression. (The proof of convergence of $\chi^{(s)}(x)$ for dispersing billiards is trivial $[1,2]$.)

Suppose that the first $n$ reflections of the trajectory of point $x$ are from neutral components of the boundary. Then $k_{1}^{(0)}(x)=k_{2}^{(0)}(x)=\ldots=k_{n}^{(0)}(x)=0$ and the corresponding continued fraction has the form

$$
x^{(s)}(x)=\frac{1}{-\left(\tau_{1}+\tau_{2}+\ldots+\tau_{n+1}\right)+\frac{1}{2 \frac{k_{n+1}^{(0)}(x)}{\cos \varphi_{n+1}(x)}+\frac{1}{-\tau_{n+2}+. \cdot}} .}
$$


Consequently by consecutive reflections of the trajectory of the line element $x \in \gamma$ from the neutral part of the boundary of the domain $Q$, the absolute value of curvature of the curve $S_{t} \gamma$ in the point $S_{t} x$ decreases inversely proportional to time until the first reflection from regular component of the boundary $\partial Q_{i}$ with nonzero curvature takes place.

We shall formulate several results from paper [5], concerning properties of the series of consecutive reflections from one and the same focusing component of constant curvature.

Since a focusing component $\Gamma_{i}^{F}$ is an arc of a circle the intervals between two successive reflections in each series of consecutive reflections from $\Gamma_{i}^{F}$ coincide, because the angle of incidence is equal to the angle of reflection. Thus the transformation $T$ for such a series is simply a rotation of the circle by the angle $\left(180^{\circ}-2 \varphi\right)$, where $\varphi$ is a constant for the series of reflections under consideration angle of incidence.

Consequently the segment of the continued fraction $x^{(s)}(x)$ corresponding to a series of reflections of length $n$ from one and the same focusing component $\Gamma_{i}^{F}$ of the boundary has the form

$$
x_{\Gamma_{l}^{F}}^{(s)}(x)=\frac{1}{-\tau_{1}+\frac{1}{\frac{4}{\tau}+\frac{1}{-\tau+\frac{1}{\frac{4}{\tau}+\ddots}+\frac{1}{-\tau+\frac{1}{\frac{4}{\tau}}}}}},
$$

where $\tau_{1}$ is the interval between the first (in this series) reflection from $\Gamma_{i}^{F}$ and the previous reflection of the trajectory of point $x$ from $\partial Q^{-}$and $\tau$ is the interval between two successive reflections from the component $\Gamma_{i}^{F}$. The fraction $\chi_{T_{i}^{F}}^{(s)}(x)$ contains $2 n$ terms.

Further in $[5$, p. 59] the following lemma is proved.

Lemma 4. An infinite continued fraction $K_{a, b}$ of the form

$$
K_{a, b}=\frac{1}{a+\frac{1}{b+\frac{1}{a+\frac{1}{b+\cdot \cdot}}}},
$$

where $a$ and $b$ are real numbers and $\alpha=a b<0$, converges if and only if $\alpha \leqq-4$, while if $\alpha=-4$, then $K_{a, b}=-\frac{b}{2}=\frac{2}{a}$. Moreover the sequence of convergents $P_{n} / Q_{n}$ is a monotone numerical sequence. 
We will mention that for the series of consequtive reflections from one and the same circle just the case $\alpha=-4$ takes place. Consider a bundle of trajectories whose curvature at any of its points $x \in \mathscr{U}_{1}$ is equal to $x^{(s)}(x)$. From the definition of the set $\mathscr{U}_{1}$ it follows that $x^{(s)}(x)$ is the curvature of this bundle (at the point $x$ ) after reflection from the boundary $\partial Q$. Then it follows from Lemma 3 that the curvature (at the same point) of this bundle at the moment before reflection from the boundary is equal to

$$
x_{-}^{(s)}(x)=x^{(s)}(x)-2 \frac{k^{(0)}(x)}{\cos \varphi_{0}(x)}
$$

In $[5, \mathrm{p} .63]$ the following proposition which is the fundamental for studying reflections from focusing part of the boundary, is proved.

Proposition 1. Suppose that on an arbitrary component $\Gamma_{i}^{F}$ of the focusing part of the boundary $\partial Q^{-}$there is incident a bundle of trajectories of zero curvature (i.e. a bundle of parallel trajectories) which has $n$ successive reflections from $\Gamma_{i}^{F}$. Then for any line element $x$ of this bundle and for any number $m(1 \leqq m \leqq n)$ the following inequalities hold:

$$
\varkappa_{-}^{(s)}\left(T^{m} x_{0}\right)<0, \varkappa^{(s)}\left(T^{m-1} x_{0}\right)>0, \varkappa^{(s)}\left(T^{m-1} x_{0}\right)>\left|\varkappa_{-}^{(s)}\left(T^{m} x_{0}\right)\right|
$$

while

$$
\lim _{\substack{m \rightarrow \infty \\ n \rightarrow \infty}} \frac{\varkappa^{(s)}\left(T^{m-1} x_{0}\right)}{\left|x_{-}^{(s)}\left(T^{m} x_{0}\right)\right|}=1 \text {, where } x_{0}=S_{\tau(x)-0} x .
$$

Less formally, in Proposition 1 it is asserted the following: if on an arbitrary focusing component $\Gamma_{i}^{F}$ falls a bundle of parallel trajectories and has a series of $n$ successive reflections from it, then after every reflection of this series this bundle becomes contracting (i.e. has a negative curvature), after which on its way between any two successive reflections from $\Gamma_{i}^{F}$ it passes through a conjugate point and comes to the boundary before the following reflection as an expanding bundle, moreover the time during which the bundle under consideration has a positive curvature (expands) is more than half of the whole time interval $(|\tau|)$ between two successive reflections from $\Gamma_{i}^{F}$. So, by successive reflections from the boundary of any focusing component (under the action of transformation $T$ ) the bundle under consideration locally expands linearly along the length of the front, and its curvature tends to a constant limit (when the number of reflections in the series tends to infinity).

\section{Proof of the Convergence of the Continued Fraction}

The following proposition holds.

Lemma 5. If a domain $Q$ satisfies conditions 1 and 2 then the continued fraction $\varkappa^{(s)}(x)$ converges for every regular point $x \in \mathscr{U}_{1}$. 
Proof. First of all we mention that if a domain satisfies condition 1 then for any regular point $x \in \mathscr{U}_{1}$ the fraction $\varkappa^{(s)}(x)$ has the following form:

$$
\begin{aligned}
& x^{(s)}(x)=\frac{1}{-\left(\tau_{1}+\tau_{2}+\ldots+\tau_{n_{1}}\right)}+\frac{1}{\mid 4 / \tau_{n_{1}+1}}+\frac{1}{-\tau_{n_{1}+1}}+\ldots+\frac{1}{\mid 4 / \tau_{n_{1}+1}} \\
& +\frac{1}{-\left(\tau_{n_{1}+k_{1}}+\ldots+\tau_{n_{1}+k_{1}+n_{2}}\right)}+\frac{1}{4 / \tau_{n_{1}+k_{1}+n_{2}+1}}+\frac{1}{-\tau_{n_{1}+k_{1}+n_{2}+1}}+\ldots
\end{aligned}
$$

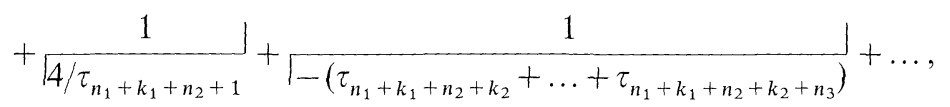

where the element $-\sum_{j=0}^{n_{m+1}} \tau \sum_{i=1}^{m}\left(n_{2}+k_{l}\right)+j$ corresponds to the $(m+1)$ th series of consecutive reflections from the neutral part of the boundary $\partial Q^{0}$ or to the jump from one focusing component to another, while a segment of continued fraction

$$
\frac{1}{\sqrt{4 / \tau} \sum_{i=1}^{m}\left(n_{l}+k_{t}\right)+n_{m+1}+1}+\frac{1}{-\tau \sum_{l=1}^{m}\left(n_{l}+k_{l}\right)+n_{m+1}+1}+\ldots+\frac{1}{\sqrt{4 / \tau \sum_{l=1}^{m}\left(n_{t}+k_{i}\right)+n_{m+1}+1}}
$$

between two following terms of such a form corresponds to the series (of the length $k_{m+1}$ ) of consecutive reflections from one and the same focusing component of the boundary $\partial Q$. Here $\left(n_{i}-1\right)$ is the length of $i$-th series of consecutive reflections from the neutral part of the boundary, $k_{i}$ is the length of $i$-th series of consecutive reflections from an arbitrary focusing component. (We will mention that if $k_{m+1}=1$ then a corresponding segment of continued fraction has the form $4 / \tau_{m+1}^{\prime}$, where, generally speaking, $\left.\tau_{m+1}^{\prime} \neq \tau \sum_{\sum_{1}}^{m}\left(n_{t}+k_{t}\right)+1\right)$. We recall that by elements of the continued fraction

$$
b_{0}+\frac{1}{\mid b_{1}}+\frac{1}{b_{2}}+\ldots
$$

we mean the numbers $b_{i}$, $(i \geqq 0)$.

For convenience we consider instead of $x^{(s)}(x)$ the continued fraction $-x^{(s)}(x)$. Then all the elements of the continued fraction $x^{(s)}(x)$ also change sign.

There are two kinds of elements in continued fraction (2): elements with odd numbers correspond to the time intervals between two reflections from the focusing part of the boundary and elements with even numbers correspond to the curvature of the boundary at the point of reflection. In what follows we shall denote a product of two successive elements $b_{2 n-1} b_{2 n}$ by $\gamma_{n}$. We mention that all the odd elements of the continued fraction $-x^{(s)}(x)$ are positive and all the even elements are negative.

Consider the recursion formulas (see [13]) for numerators and denominators of convergents $P_{n} / Q_{n},(n \geqq 3)$

$$
P_{n}=b_{n} P_{n-1}+P_{n-2}, \quad Q_{n}=b_{n} Q_{n-1}+Q_{n-2} .
$$


From them it is easy to get the relations $(n \geqq 5)$

$$
\begin{aligned}
& P_{n}=\left(b_{n} b_{n-1}+\frac{b_{n}}{b_{n-2}}+1\right) P_{n-2}-\frac{b_{n}}{b_{n-2}} P_{n-4}, \\
& Q_{n}=\left(b_{n} b_{n-1}+\frac{b_{n}}{b_{n-2}}+1\right) Q_{n-2}-\frac{b_{n}}{b_{n-2}} Q_{n-4} .
\end{aligned}
$$

For the fraction $-\chi^{(s)}(x)$ Formula (4) becomes

$$
P_{n}=\left(\gamma\left[\frac{n}{2}\right]+1+2\right) P_{n-2}-P_{n-4}, Q_{n}=\left(\gamma_{\left[\frac{n}{2}\right]+1}+2\right) Q_{n-2}-Q_{n-4} .
$$

We write down the first four convergents:

$$
\begin{aligned}
& \frac{P_{1}}{Q_{1}}=\frac{1}{b_{1}} ; \frac{P_{2}}{Q_{2}}=\frac{b_{2}}{b_{1} b_{2}+1} ; \frac{P_{3}}{Q_{3}}=\frac{b_{2} b_{3}+1}{b_{3}\left(b_{1} b_{2}+1\right)+b_{1}} ; \\
& \frac{P_{4}}{Q_{4}}=\frac{b_{4}\left(b_{2} b_{3}+1\right)+b_{2}}{b_{4}\left[b_{3}\left(b_{1} b_{2}+1\right)+b_{1}\right]+b_{1} b_{2}+1} .
\end{aligned}
$$

Hence, using (4), (5) it follows that $\operatorname{sgn} Q_{2 n+1}=-\operatorname{sgn} Q_{2 n-1}$ and $\operatorname{sgn} Q_{2 n+2}$ $=-\operatorname{sgn} Q_{2 n}$. In fact, from the conditions 1,2 it follows that for all $\gamma_{k}: \gamma_{k}+2 \leqq-2$. It is easy to get required assertion from Formulae (4), (6) and condition 2.

Since we have the following recursion formulae (see [13])

$$
\frac{P_{2 k+1}}{Q_{2 k+1}}-\frac{P_{2 k-1}}{Q_{2 k-1}}=-\frac{b_{2 k+1}}{Q_{2 k-1} Q_{2 k+1}}, \quad \frac{P_{2 k}}{Q_{2 k}}-\frac{P_{2 k-2}}{Q_{2 k-2}}=\frac{b_{2 k}}{Q_{2 k-2} Q_{2 k}}
$$

we get that the sequences of even and odd convergents are increasing. Now using the well known (see [13]) recursion relation

$$
\frac{P_{n+1}}{Q_{n+1}}-\frac{P_{n}}{Q_{n}}=(-1)^{n} \frac{1}{Q_{n} Q_{n+1}}
$$

and the property of interchanging of signs of denominators in the sequences of even and odd convergents we proved earlier, we get that the sequence of all convergents is increasing.

From relations (4) and (6) it follows that

$$
\left|Q_{2 n+1}\right|>\tilde{C}_{1} n,\left|Q_{2 n}\right|>\tilde{C}_{2} n,
$$

where $\tilde{C}_{1}, \tilde{C}_{2}$ are some constants.

Consequently

$$
\left|\varkappa^{(s)}(x)\right| \leqq\left|\frac{P_{1}}{Q_{1}}\right|+\left|\frac{P_{2}}{Q_{2}}-\frac{P_{1}}{Q_{1}}\right|+\left|\frac{P_{3}}{Q_{3}}-\frac{P_{2}}{Q_{2}}\right|+\ldots=\left|\frac{1}{Q_{1}}\right|+\sum_{n=1}^{\infty} \frac{1}{\left|Q_{n} Q_{n+1}\right|}<\infty .
$$

Therefore the sequence of convergents $\left\{P_{n} / Q_{n}\right\}$ has a limit, as it is monotone and bounded, which completes the proof of the lemma.

It is not difficult to see that the function $x^{(s)}(x)$ is continuous almost everywhere. In fact, suppose that the positive semitrajectory of the point $x$ does not contain singular points of the boundary $\partial Q$. According to the proof we gave 
earlier the continued fraction $x^{(s)}(x)$ converges, therefore for every $\varepsilon>0$ one can find a number $n=n(\varepsilon)$ such that $\left|x^{(s)}(x)-x_{n}^{(s)}(x)\right|<\frac{\varepsilon}{3}$, where $x_{n}^{(s)}(x)$ is the $n$-th convergent. Owing to the fact that the semitrajectory under consideration contains only regular points of the boundary there exists a neighbourhood $U \ni x$, such that for every point $y \in U$ the following inequality holds: $\left|x_{n}^{(s)}(x)-x_{n}^{(s)}(y)\right|<\frac{\varepsilon}{3}$. Finally, from conditions 1,2 and from the proof of Proposition 1 it follows that for all points $z \in \mathscr{U}_{1}^{-}$whose trajectories contain no singular points the inequality $\left|x^{(s)}(z)-x_{n}^{(s)}(z)\right|<\frac{\varepsilon}{3}$ holds, where $n$ depends only on $\varepsilon$ and not on $z$. Therefore our assertion is true.

\section{Main Theorem}

Now we shall construct two vector fields $\mathfrak{U}^{(s)}$ and $\mathfrak{U}^{(u)}$ in the phase space $M$. In a neighbourhood of point $x=(q, \varphi)$ let us choose coordinates $q_{1}, q_{2}$, and $\varphi$ so that the angle $\varphi$ is computed from the axis $q_{1}$. The components of the tangent vector of the field $\mathfrak{U}^{(s)}$ at the point $x$ have the form $\left(-\sin \varphi, \cos \varphi, \varkappa^{(s)}(x)\right)$. From Lemmas 1 and 2 it follows that the vector field $\mathfrak{u}^{(s)}$ is invariant under the action of the flow $\left\{S_{t}\right\}$ in the following sense: if $\left\{\tilde{S}_{t}\right\}$ is the one-parameter group of transformations of the tangent space to $M$ onto itself induced by our flow, then the straight line $\mathfrak{U}^{(s)}(x)$, which has the direction of the vector field $\mathfrak{U}^{(s)}$ at the point $x$, is carried under the action of $\tilde{S}_{t}$ into the same line $\mathfrak{U}^{(s)}\left(S_{t} x\right)$.

We project the vector field $\mathfrak{U}^{(s)}$ onto the boundary $\mathscr{U}_{1}$. If $x=(r, \varphi) \in \mathscr{U}_{1}$, we set $\frac{d \varphi}{d r}=-\varkappa^{(s)}(x) \cos \varphi(x)-k^{(0)}(x)$. The vector field $\mathfrak{U}_{T}^{(s)}$ which we obtain has an invariance property of the same type relative to the transformation $T$.

To construct the vector field $\mathfrak{H}^{(u)}$ we consider the involutive automorphism $I$ of the space $M$, which transforms the line element $x \in M$ into the line element with the same support and opposite direction. We set $x^{(u)}(x)=-x^{(s)}(I x)$. It is easy to see that the vector field defined by means of the function $x^{(u)}(x)$ will also be invariant. Its projection on the boundary $\mathscr{U}_{1}$ has the form

$$
\frac{d \varphi}{d r}=-x^{(u)}(x) \cos \varphi(x)+k^{(0)}(x) .
$$

It follows from Lemmas $1-3$ and 5 that for $\mathfrak{U}^{(s)}$ we have: $\frac{d \varphi}{d r}>0$ if $\pi(x) \in \partial Q^{-}$. For the vector field $\mathfrak{U}^{(u)}$ the opposite inequality holds.

We remind that by a locally contracting transversal fiber (l.c.t.f.) of the point $x$, in some neighbourhood $U$ of $x$, is meant a curve in $U$ consisting of points $y \in U$ for which $d\left(S_{t} x, S_{t} y\right) \rightarrow 0$ as $t \rightarrow \infty$. Letting $t$ tend to $-\infty$, we get the definition of a locally expanding transversal fiber (l.e.t.f.). The definition of locally transversal fibers for the transformation $T$ is the same as for $\left\{S_{t}\right\}$ except that in place $S_{t}$ one must write $T^{n}$ and let $n$ tend to $\pm \infty$. Such fibers play a central role in studying of ergodic properties of classical dynamical systems, such as Anosov systems, partially hyperbolic systems, dispersing billiards and so on (see [14-16]). 
From Lemmas 1-3 describing the reflection from the boundary it is not difficult to deduce that locally transversal fibers of the transformation Tare (if they exist for the billiards we study) just the trajectories of the vector fields $\mathfrak{U}_{T}^{(s)}$ and $\mathfrak{U}_{T}^{(u)}$.

We will come now to the proof of the main theorem which was formulated in $\S 1$. We shall not give here all the proofs of this theorem since it takes too much place. Besides, many stages of that proof repeat almost step by step the proof of the existence of the $K$-property for other classes of billiards (see papers $[1,2,5]$ and also the lectures [16]).

We shall prove in detail only facts that are directly connected with the specific class of billiards we study, i.e. with geometrical properties of corresponding domains.

In what follows we shall examine the derived automorphism $T$, unless we state otherwise. The main fact which must be verified in the proof of ergodicity of an arbitrary class of billiards is that for almost every point $x$ of the phase space $\mathscr{U}_{1}$ the local fiber tangent in the point $x$ to the vector field $\mathfrak{u}^{(s)}$ (or $\mathfrak{U}^{(u)}$ ) is in fact contracting (or expanding). We shall point out the mechanism which generates contraction (expansion) for the class of billiards under consideration.

Let the point $x_{0} \in \mathscr{U}_{1}$ be fixed, and set $x_{i}=T^{i} x_{0}$. We assume that $x_{i} \notin S$ for $i=0,1,2, \ldots$, i.e. the negative semitrajectory of the point $x_{0}$ has no intersections with the singular part of the boundary. We introduce the following quantities:

$$
d_{i}=d\left(x_{i}\right), \cos \varphi_{i}=\cos \varphi\left(x_{i}\right), \tau_{i}=\tau\left(x_{i}\right), x_{i}^{(s)}=x^{(s)}\left(x_{i}\right) .
$$

Then the (local) coefficient of contraction $\lambda_{i}^{(s)}\left(x_{0}\right)$ under the action of the transformation $T$ at the point $x_{i-1} \in \mathscr{U}_{1}$ equals $\frac{\left|\tau_{i}\right|-1 / x_{i-1}^{(s)}}{1 / x_{i-1}^{(s)}}$ if $\pi\left(x_{i-1}\right) \in \partial Q^{-}$and the corresponding local bundle of trajectories during a time $\left|\tau_{i}\right|$ passed through a conjugate point. In fact, this equality follows immediately from considerations of $\S 2$ (a more detailed exposition can be found in $\S 3$ of the paper [5]). If the local bundle of trajectories under consideration during the motion from $x_{0}$ until $x_{i}$ did not pass through a conjugate point, the local coefficient of contraction equals $\left(1+\chi_{i-1}^{(s)}|\tau|\right)^{-1}$ (see Lemma 1). However observe that both in the first and in the second case, we are considering only contraction along the $r$-axis in phase space $\mathscr{U}_{1}$. As to the change of "length" of the local bundle along the angle coordinate $\varphi$, it is obvious that it can take place only under reflection from the focusing part of the boundary.

Therefore it is convenient to introduce a new special representation of the flow $\left\{S_{t}\right\}=(\tilde{T}, \tilde{\tau})$. The transformation $\tilde{T}$ acts on the set $\tilde{\mathscr{U}}_{1}^{-}$and for any point $x \in \tilde{\mathscr{U}}_{1}^{-}$the number $\tilde{\tau}(x)$ is the nearest negative moment of reflection of the trajectory of the point $x$ under the action of the flow $\left\{S_{t}\right\}$ from the boundary at a point belonging also to the set $\tilde{\mathscr{U}}_{1}^{-}$. Thus $\tilde{T} x=S_{\bar{\tau}(x)-0} x$, where $x \in \tilde{\mathscr{U}}_{1}^{-}$.

First of all we shall show that almost every trajectory of the flow $\left\{S_{t}\right\}$ passes through the set $\tilde{\mathscr{U}}_{1}^{-}$. We mention that almost every trajectory of the flow under consideration contains reflections from the focusing part $\partial Q^{-}$of the boundary of the domain $Q$. In fact, for an arbitrary polygon each trajectory of corresponding billiard comes up as close as one wants to one of its vertexes (see [11]). The reguired assertion follows easily now from the geometrical properties of the boundary of the domain under consideration and from the results of $\S 3$. 
Further, one can get from the conditions of the theorem that for almost all trajectories reflections from the boundary of the domain $Q$ take place sufficiently often at the points of the set $\tilde{\mathscr{U}}_{1}^{-}$. In fact, according to the Lemma of Borel-Cantelli from the convergence of the series $\sum_{n=1}^{\infty} \mu\left(A_{n}^{\alpha}\right)$ one can get that the trajectory of almost every point $x \in \mathscr{U}_{1}^{-}$hits the collection of sets $A_{n}^{\alpha}$ only for a finite number of values of $n$.

Consequently for almost every point $x \in \mathscr{U}_{1}$ there exist positive numbers $C(x)<\infty, \lambda(x)<1, \alpha_{1}<1$ such that for all $m>0$ the following inequality holds

$$
\prod_{i=1}^{m}\left(\lambda_{i}^{(s)}(x)\right)^{-1} \leqq C(x)(\lambda(x))^{m(1-\alpha)} .
$$

Since the trajectory of almost every point hits the collection of sets $A_{n}^{\alpha}$ only a finite number of times, for a set of points $x \in \mathscr{U}_{1}$ of full measure the length of the series beginning from the $k$-th reflection and consisting of consecutive reflections from the complement of the set $\tilde{\mathscr{U}}_{1}^{-}$does not exceed $\tilde{C} k^{\alpha_{1}}$, where $\tilde{C}$ is a constant, $k>k_{0}(x), \alpha_{1}<1$. Let us now consider the segment of length $m$ of the trajectory of the point $x \in \mathscr{U}_{1}$ beginning from the first reflection of this point from the boundary. Then, according to the conditions of theorem within such a trajectory segment not less than $\bar{C} m^{1-\alpha_{1}}$ reflections from the boundary at points of the set $\tilde{\mathscr{U}}_{1}^{-}$must take place. Inequality (7) follows from this and it means that for a set of whole measure contraction (expantion under the action of $T^{-1}$ ) along the direction of the vector field $\mathfrak{U}_{T}^{(s)}$ does in fact take place.

This allows us to construct l.c.t.f. and l.e.t.f. for almost every point $x \in \mathscr{U}_{1}$. The process of construction of these fibers is a variant of the proof of HadamarPerron's theorem for manifolds (see $[1,14]$ ). After which the whole leaves of the contracting and expanding foliations can be constructed in the usual way. These leaves possess singularities (cusps points), corresponding to trajectories which fall on the points of intersection of the regular components of the boundary. One can work out the following stages of the demonstration of the $K$-property for our billiards, in analogy with paper [5] (see also [1,2]), with some minor modifications, $B$-property can be concluded from $K$-property just in the same way as for dispersing billiards (see [17]).

\section{Some Examples of Applications of the Main Theorem}

In this paragraph we shall consider several classes of domains, which satisfy the conditions of Theorem 1 . We shall suppose that the geometrical properties we reguired are always satisfied.

\section{1. "Stadion" 1,2 and Its Modifications}

In this section we consider domains with boundaries consisting of two neutral, $\bar{\Gamma}_{1}$ and $\bar{\Gamma}_{2}$, and two focusing, $\hat{\Gamma}_{1}$ and $\hat{\Gamma}_{2}$, components with the end-points of every

1 The term "stadion" was introduced independently and almost simultaneous by D. V. Anosov and Ya. G. Sinai

2 In the english literature the term "billiard on a football field" can be also met 


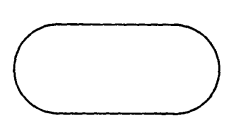

a

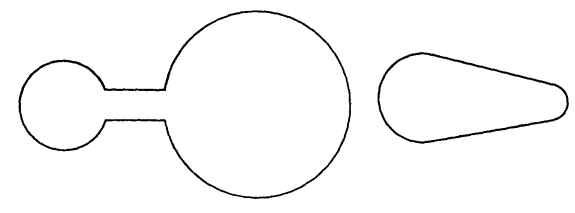

C

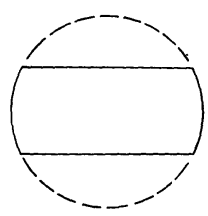

d

Fig. 3

focusing component belonging to the neutral part of the boundary. Some domains of this type are represented in Fig. 3. The domain which is represented in Fig. 1a also belongs to this class.

The most popular example - the "stadion" - a region whose boundary consists of two identical half-circles and two parallel segments, is represent in Fig. 3a. It is the first example of a convex region inside which the billiard is ergodic [8].

We now show that for the class of domains considered in this section all the conditions of the main theorem are satisfied. Let us consider first of all what is in our case the set $\tilde{\mathscr{U}}_{1}^{-}$. It is clear that the line element $x \in \tilde{\mathscr{U}}_{1}^{-}$if $\pi(T x) \in \hat{\Gamma}_{i}$ and $\pi(x)$ belongs to the neutral part of the boundary (excluding the case where $\hat{\Gamma}_{1}$ and $\hat{\Gamma}_{2}$ are arcs of one and the same circle, Fig. 3). In the latter case both here and for classes of regions considered below the proof requires some obvious changes and we shall not consider it separately.

Now let us see in which cases a trajectory does not fall on the set $\tilde{\mathscr{U}}_{1}^{-}$for a long time. It is clear that this can take place when a trajectory reflects from one and the same focusing component of the boundary during a long time, or has very long series of consecutive reflections from the neutral part of the boundary of the domain. The first possibility occurs when the corresponding segment of the trajectory under consideration goes near to a certain periodic trajectory of the billiard inside the circle which contains this focusing component, if the component is larger than a half-circle (Fig. 1), or when the trajectory is almost tangent to the boundary, i.e. times between two successive reflections are very small.

We shall consider the first case, when the arc $\hat{\Gamma}_{i}$ is larger than a half-circle. We shall mention that before the first reflection from $\hat{\Gamma}_{i}$ in the series under consideration the trajectory must intersect with the complementary arc $O_{\hat{\Gamma}_{i}} \backslash \hat{\Gamma}_{i}$. Let us consider the set of all line elements with one and the same support $q \in O_{\hat{\Gamma}_{i}}$. This set is a half-circle which we shall denote by $O_{q}$.

Let $B_{n}=\left\{x \in O_{q}: \pi\left(T_{j}(x)\right) \in \hat{\Gamma}_{i}\right.$ for $\left.1 \leqq j \leqq n\right\}$. It is easy to see, that $v_{q}\left(B_{n}\right)<C^{\prime} / n$, where $v_{q}$ is the regular Lebesgue measure on $Q_{q}$ and $C^{\prime}$ is a constant. Now let $D_{n}$ be a subset of $\pi^{-1}\left(O_{\hat{\Gamma}_{i}} \backslash \hat{\Gamma}_{i}\right)$, such that $D_{n}=\left\{x: S_{\tau(x)-0} x=x_{0}, T^{k} x_{0} \in \hat{\Gamma}_{i}\right.$ for all $\left.1 \leqq k \leqq n\right\}$. Then $\mu_{O_{\hat{\Gamma}}}\left(B_{n}\right)<\mathrm{C}^{\prime \prime} / n$, where $\mu_{O_{\hat{\Gamma}_{i}}}$ is a measure on $O_{\Gamma_{i}}$ induced by the measure $\mu$ on $M$ and $C^{\prime \prime}$ is a constant. Therefore a measure of the set of those trajectories which spread inside $O_{\hat{\Gamma}_{\imath}}$ on an angle larger than $2 \pi n$ is not more than $C^{\prime} C^{\prime \prime} / n^{2}$. So the condition of the main theorem for the set of such trajectories is satisfied. One can get analogous inequality for the measure of the set of trajectories which go along the boundary of $\hat{\Gamma}_{i}$. In fact, since $d v=-\operatorname{const} \cos \varphi d r d \varphi$, the measure of the set of trajectories which have more than $n$ successive reflections from $\hat{\Gamma}_{i}$ and are quasi-tangent is less than $\hat{C} / n^{2}$ because the angle between any segment of such a 


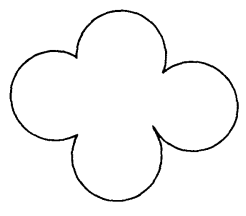

a

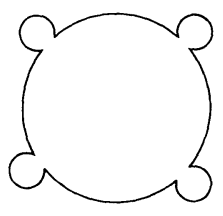

b

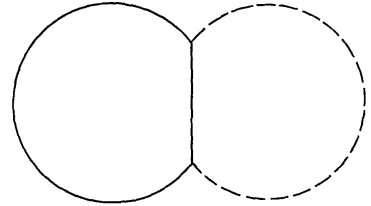

C

Fig. 4

trajectory and a vector normal to the boundary is close to $\pi / 2$. Consequently, the condition of the main theorem is satisfied for this set of trajectories.

Let us consider at last a trajectory which for a long time undergoes reflections on the neutral part of the boundary. It is clear that this can occur only in the case when neutral components of the boundary are parallel and the trajectory under consideration reflects from them with a very small angle $\varphi$.

Let $\delta>0$ and $E_{n, i}^{\delta}=\left\{x=(r, \varphi): \pi(x) \in \partial Q^{0},|\pi-\varphi|<1 / n^{\delta},\left|r-r_{i}^{\prime}\right|<1 / n^{\delta}\right\}$, where $r_{i}^{\prime}$ $(i=1,2,3,4)$ are $r$-coordinates of the singular points of the boundary. It is clear that $v\left(E_{n, i}^{\delta}\right)=\mathrm{const} / n^{2 \delta}$. Let $\delta=\frac{1}{2}+\theta$, where $\theta>0$. Then the series $\sum_{n=1}^{\infty} v\left(E_{n, i}^{\delta}\right)$ converges. Let us consider to the following important circumstance : if in a series of consecutive reflections from the neutral components the angle of incidence is small and equals $\varphi_{0}$, then after the end of this series and after the following reflection (or series of reflections) from any focusing component of the boundary the angle of incidence in the nearest following series of reflections from the neutral part of the boundary will be strongly larger than $\varphi_{0}$ (for example, in the case of stadion $\varphi_{1} \geqq 2 \varphi_{0}$, in the nearest following series of "neutral" reflections $\varphi_{2} \geqq 4 \varphi_{0}$ and so on). Therefore for a long time after a series of reflections from the neutral components in furthered, only shorter series of reflections from the neutral components take place. So all the conditions of the Theorem 1 are satisfied for the class of billiards under consideration in this section and the corresponding billiards generate dynamical systems isomorphic to Bernoullian flows.

\section{2. "Flowerlike" Regions and Circle Segments}

The domains which are considered in this section have only focusing components in their boundaries. Billiard in a circle segment is actually a special case of such billiards (Fig. 4c) as it is equivalent to a billiard in a region which appears after reflection of the arc of a circle relative to the chord (a neutral component). This example is important also as it most clearly explains the necessity of condition 2 introduced in $\S 1$. In fact, condition 2 in our case means that the corresponding region is a circle segment with a central angle more than $\pi$ (i.e. contains a halfcircle). It is not difficult to prove, that if a circle segment is not more than half a circle the corresponding billiard is nonergodic, i.e. for a half-circle it is obvious and in the other cases there exists a stable periodic trajectory of period 2 (see Fig. 2b).

The proof that the conditions of our main theorem are satisfied is simpler in this case than in the case of the preceding section, since here the third "bad" case is impossible. 


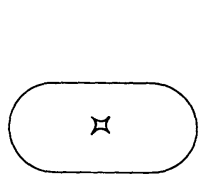

a

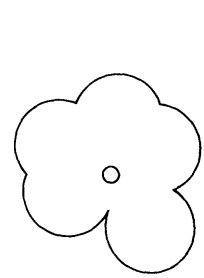

b

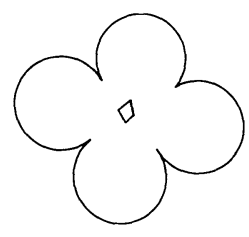

C

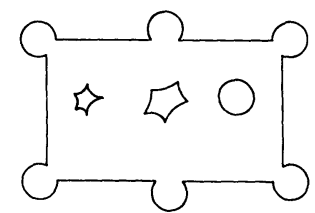

d

Fig. 5

In concluding this paragraph we mention, that there exist other classes of regions satisfying the conditions of the main theorem. In particular one can construct such domain by "putting together" pieces of different domains of the type considered in Sects. 5.1 and 5.2. However, one can consider non singly connected domains, i.e. from the interior one can exclude several subdomains and the resulting billiards will still have the $B$-property (one must look for preserving of the properties 1,2 and conditions of the main theorem). Examples of such domains are represented in Fig. 5.

We shall mention that the billiard in the region represented in Fig. $5 b$ does not belong formally to the class considered in this paper since the boundary of the subregion excluded is dispersing. But from the results of paper [5] and of this paper it follows immediately that the billiard in this region is a $B$-system.

\section{Multidimensional Generalizations and Consulding Remarks}

Consider now any domain $Q$ with peacewise smooth boundary in Euclidian space $R^{n}$ or on an $n$-dimensional torus with Euclidian metric. The billiard in $Q$ is defined in the same way like in the two dimensional case. The definitions of equipments of unit normals of submanifolds of codimension one are defined analogously. Every such equipment is a section of the unit tangent bundle. When the equipment is defined one can introduce the operator of the second quadratic form of the submanifold under consideration. The corresponding local bundle of trajectories is in this case expanding or contracting if the operator of the second quadratic form defined at the given point is positive or negative respectively.

One can define classes of billiards in multidimensional domain which correspond to the classes we considered in this paper. First of all we make a simple but very important remark : every trajectory of a billiard inside a sphere is flat and moves along the circumference of a maximal circle of this sphere. The assertion of this remark follows from the fact that three arbitrary points of the space define a plane and from the law of reflection of billiard trajectories from the boundary.

Therefore, for example, billiards in regions with boundaries consisting only of spheres possess the $B$-property if conditions analogous to condition 1,2 and to the conditions of the main theorem hold. In particular, a billiard in a spherical segment is a Bernoullian flow if this segment contains a half-sphere.

One can show also, that billiards in multidimensional domains which appear under rotation of regions of Sect. 5.1. around the natural axis possess the $B$ - 
property. In particular from the stadion we get a region with boundary consisting of a cylinder and two half-spheres.

In conclusion we shall make some remarks concerning the connection between the smoothness of the boundary of the domain and the statistical properties of the dynamical system generated by the corresponding billiard. First of all we mention that in all examples considered in this paper the smoothness of the boundary of the domain $Q$ was not more than $C^{1}$. It is easy to see that if the boundary $\partial Q$ is a curve of the class $C^{k}$ the corresponding derived automorphism $T$ of the flow generated by billiard belongs to the class $C^{k-1}$. However in the paper of Lazutkin [7] it was shown that a billiard inside a sufficiently smooth $\left(C^{k}, k \geqq 553\right)$ convex curve in the two - dimensional plane possess infinitely many caustics which fill in a set of positive measure. The new approach of Rüssmann [8] to the problem of small denominators allows to relax the conditions in Lazutkin's theorem from $C^{553}$ bis $C^{8}$. It is obvious that the existence of a single caustic entails nonergodicity of corresponding billiard. In this connection the following question arises: does there exist for a generic sufficiently smooth convex curve in the two-dimensional plane an ergodic component of positive measure of the corresponding billiard? [It is natural also to mention here the paper [19], where the passage from an integrable system (billiard inside a circle) to a stochastic system ("stadion") was studied with the help of modelling on electronic machine. However every region in the interval between these two extremes has a boundary of class $C^{1}$ only.] For an arbitrary smooth curve this assertion is not right. For example, it is known [20] that the billiard in ellips is a completely integrable system. The theorem by Dvorin and Lazutkin on the existence of infinitely many periodic trajectories of hyperbolic type for billiards inside a generic convex smooth curve in the plane can be considered as an indirect support of this conjecture [21].

Acknowledgements. The author thanks Ya. G. Sinai for valuable discussions and C. Boldrighini for help in translation into English.

\section{References}

1. Sinai, Ya.G. : Dynamical systems with elastic reflections. Ergodic properties of dispersing billiards. Usp. Mat. Nauk 25, 141-192 (1970); Russ. Math. Surv. 25, 137-189 (1970)

2. Bunimovich, L.A., Sinai, Ya.G.: On a fundamental theorem in the theory of dispersing billiards. Mat. Sb. 90, 415-431 (1973); Math. USSR Sb. 19, 407-424 (1973)

3. Arnold, V.J.: Small denominators and problems of stability of motion in classical and celectial mechanics. Usp. Mat. Nauk 18, 91-192 (1963); Russ. Math. Surv. 18, 85-191 (1963)

4. Hopf, E.: Statistik der Lösungen geodätischer Probleme vom unstabilen Typus. II. Math. Ann. 117, 590-608 (1940)

5. Bunimovich, L. A. : On billiards close to dispersing. Mat. Sb. 94, 49-73 (1974); Math. USSR Sb. 23, 45-67 (1974)

6. Birkhoff, G.D. : Dynamical systems. Am. Math. Soc. Colloq. Dubl., Vol. 9. Providence, R.I.: AMS 1927 ; rev. ed. 1966

7. Lazutkin, V.F.: The existence of coustics for the billiard problem in a convex domain. Izv. A.N. SSSR, ser matem 37, 186-216 (1973); Math. USSR Izv. 37, 186-216 (1973)

8. Bunimovich, L.A.: On the ergodic properties of some billiards. Funkt. Anal. Jego Prilog. 8, 73-74 (1974); Funct. Anal. Appl. 8, 73-74 (1974)

9. Rohlin, V.A.: Selected topics from the metric theory of dynamical systems. Usp. Mat. Nauk 4, 57-128 (1949); English transl.: Am. Math. Soc. Transl. (2) 49, 171-240 (1965) 
10. Zemlyakov, A.N., Katok, A.B.: The topological transitivity of billiards in polygons. Mat. zametki 18, 291-301 (1975)

11. Boldrighini, C., Keane, M., Marchetti, F.: Billiards in polygons. Preprint, Univ. di Camerino, Italy (1977)

12. Ornstein, D.S.: An example of a Kolmogorov auromorphism that is not a Bernoulli shift. Adv. Math. 10, 49-62 (1973)

13. Hovanskij, A.N.: Application of continued fractions and their generalizations to problems in approximate theory. Moscow: Gittl 1956; English transl.: Groningen: Noordhoff 1963

14. Anosov, D.V.: Geodesic flows on closed Riemannian manifolds of negative curvature. Trudy Mat. Inst. Steklov 90, 3-209 (1967); Proc. Steklov Inst. Math. 90, 1-227 (1969)

15. Brin, M.J., Pesin, Ya. B. : Partially hyperbolic dynamical systems. Izv. A.N. SSSR, ser. matem. 38, 170-212 (1974); Math. USSR Izv. 7, 185-227 (1973)

16. Gallavotti, G.: Lectures on billiards. In: Dynamical systems, theory and applications. Lecture notes in physics, Vol. 38. Berlin, Heidelberg, New York: Springer 1975

17. Gallavotti, G., Ornstein, D. S. : Billiards and Bernoulli schemes. Commun. math. Phys. 38, 83-101 (1974)

18. Rüssmann, H.: Kleine Nenner. I. Über invariante Kurven differenzierbarer Abbildungen eines Kreisrings. Nachr. Acad. Wiss., Göttingen, Math. Phys. K1. II, 67-105 (1970)

19. Bennettin, G., Strelcyn, J.M.: Numerical experiments on a billiard stochastic transtition and entropy. Preprint, Univ. Paris-Nord, France (1977)

20. Sinai, Ya. G.: Introduction to ergodic theory. Math. notes, Vol. 18. Princeton: Princeton University Press 1976

21. Dvorin, M.M., Lazutkin, F.F.: Existence of infinitely many elliptic and hyperbolic periodic trajectories for convex billiard. Funkt. Anal. Jego Prilog. 7, 20-27 (1973); Funct. Anal. Appl. 7, 103-112 (1974)

Communicated by J. L. Lebowitz

Received August 1, 1978 